pragMATIZES - Revista Latino Americana de Estudos em Cultura

Artigos 


\title{
Desterritorialización, cultura internacional-popular e identidad en el cine: El caso del western chileno "Sal"
}

\author{
Deterritorialization, international-popular culture, and identity on \\ cinema: The case of the Chilean western 'Sal'
}
Deterritorialização, cultura internacional-popular e identidade no cinema: o caso da western chileno "Sal"

\author{
Pablo Matus'
}

\author{
Palabras clave: \\ Globalización \\ Desterritorialización \\ Cultura \\ Identidad \\ Cine
}

\section{Resumen:}

El presente artículo dialoga con la tradición de estudio cultural asociado a la producción de bienes simbólicos, particularmente respecto de las formas de representación de la identidad local latinoamericana presentes en objetos mediales de distribución globalizada. En este caso se tomará como pieza de análisis el filme chileno "Sal" (2012), que se declara como perteneciente al género del western y posee un argumento acorde con el canon reconocible al mismo, pero fue dirigida por un argentino (Diego Rougier), protagonizada por un español (Fele Martínez) y chilenos (Patricio Contreras y Javiera Contador, entre otros), y cuenta con locaciones en España y Chile. Para enfrentar el análisis se emplean principalmente conceptos del sociólogo brasileño Renato Ortiz respecto de la globalización y la mundialización, así como de lo que lo que él llama desterritorialización y cultura internacional-popular. 


\begin{abstract}
:
This paper propose a dialogue with the tradition of cultural studies applied to the production of symbolic goods, particularly about the forms of representation of Latin American identity on media objects of globalized distribution. In this case the Chilean film "Sal" (2012) will be taken as a piece of analysis, which is declared as belonging to the western genre and has an argument according to the recognizable canon,but was directed by an Argentine (Diego Rougier), starring a Spanish (Fele Martínez) and Chileans (Patricio Contreras and Javiera Contador, among others), and has locations in Spain and Chile. For the analysis, concepts of the Brazilian sociologist Renato Ortiz regarding globalization are used, as well as what he calls deterritorialization and international-popular culture.
\end{abstract}

\section{Keywords:}

Globalization

Deterritorialization

Culture

Identity

Cinema

\section{Palavras chave:}

Globalização

Desterritorialização

Cultura

Identidade

Cinema

\section{Resumo:}

Este artigo propõe um diálogo com a tradição de estudos culturais aplicados à produção de bens simbólicos, particularmente sobre as formas de representação da identidade latino-americana em objetos de mídia de distribuição globalizada. Neste caso, o filme chileno "Sal" (2012) será tomado como uma análise, que é declarado como pertencente ao gênero western e tem um argumento de acordo com o cânon reconhecível, mas foi dirigido por um argentino (Diego Rougier), estrelado por um espanhol (Fele Martínez) e chilenos (Patricio Contreras e Javiera Contador, entre outros), e tem locações na Espanha e no Chile. Para a análise, são utilizados conceitos do sociólogo brasileiro Renato Ortiz sobre globalização, bem como o que ele chama de desterritorialização e cultura internacional-popular. 


\section{Desterritorialización, cultura internacional-popular e identidad en el cine: El caso del western chileno "Sal"}

\section{Introducción}

En las últimas décadas el concepto de globalización se ha impuesto como uno de los tópicos principales en el debate académico y profesional de diversos campos, como la sociología (p.e. BAUMAN, 2010; LUHMANN, 1997; RITZER, 2007), la politología (p.e. CASTLES; DAVIDSON, 2000; INGLEHART, 2000; OUGAARD, 2004), la administración (p.e. JENSEN; SANDSTRÖM, 2011) e incluso las ciencias ambientales (KÜTTING, 2004), solo por mencionar algunos. Aunque parece un asunto nuevo, de hecho el fenómeno ha sido representado mediante metáforas desde hace tiempo, como ocurrió con las nociones de aldea global (MCLUHAN; POWERS, 1995 [1989]) y sociedad de la información (CASTELLS, 1999).

A partir de la mayoría de estas lecturas es posible advertir que el desarrollo tecnológico ha sido considerado generalmente como la variable clave, desde la masificación de medios como la radio y la televisión, a principios del siglo pasado, hasta el auge contemporáneo de internet, los medios sociales (p.e. YouTube, Twitter) y los aparatos portátiles para la interconexión (p.e. teléfonos móviles). En consecuencia, la tecnología ha sido vista casi siempre como responsable de este cambio en la sociedad, particularmente en las formas de relación, de producción y de trabajo (CHAREONWONGSAK, 2002).

Pese a esto, los efectos de la globalización no se reducen a los económicos y tecnológicos. Se ha estudiado también su impacto en la cultura misma a través de la producción y distribución global de bienes simbólicos (LIZARDO, 2008), es decir, productos intangibles de consumo y efecto esencialmente cultural, como los imaginarios producidos y difundidos por los medios masivos (DEMONT-HEINRICH, 2011; KRAIDY, 2002). Todo esto se advierte por ejemplo en los ahora clásicos estudios culturales británicos, cuando se pensó en los efectos de la televisión (WILLIAMS, 1974) y el cine (HALL, 1989) y su vínculo con la generación de nuevas identidades (HALL; JEFFERSON, 1993).

Esa tradición de estudio cultural asociado a la producción y distribución de bienes simbólicos motiva el presente artículo, que busca indagar en las formas de representación de la identidad local latinoamericana, como conjunto o como expresiones de nacionalidad, presentes en objetos mediales de producción y distribución globalizada, sobre todo cuando dicho producto pertenece a un género que a su vez se identifica con patrones culturales tradicionalmente distintos de lo latinoamericano.

En este caso se tomará como pieza de análisis el filme chileno "Sal" (2012), que se declara como perteneciente al género del western y posee un argumento acorde con el canon reconocible al mismo (p.e. locación en exteriores desérticos y conflictos que se resuelven mediante el uso de armas de fuego), pero fue dirigida por un argentino (Diego Rougier), protagonizada por un español (Fele Martínez) y chilenos (Patricio Contreras, Javiera Contador y Sergio Hernández, entre otros), y cuenta con locaciones en España y Chile.

Para enfrentar el análisis desde una perspectiva latinoamericana se emplearán principalmente conceptos del sociólogo brasileño Renato Ortiz respecto de la globalización y la mundialización, así como de lo que lo que él llama desterritorialización y cultura internacional-popular. 


\section{Marco teórico}

\subsection{Globalización desde América Latina}

Entre las lecturas que desde América Latina se han hecho de la globalización hay dos que destacan por su pertinencia al tema de este artículo: la del argentino Néstor García Canclini y la del brasileño Renato Ortiz.

Uno de los principales aportes de García Canclini al estudio de la globalización" es la idea de hibridación, que remite a procesos socioculturales en los que estructuras o prácticas discretas, que existían en forma separada, se combinan para generar nuevas estructuras, objetos y prácticas (GARCÍA CANCLINI, 2001 [1990], p. 15). Para él, la globalización puede caracterizarse, especialmente en la realidad latinoamericana, por el fomento de nuevas prácticas y estructuras que no necesariamente dan cuenta de nuevas identidades autónomas sino más bien de realidades heterogéneas, que desde este punto de vista constituyen hibridaciones interculturales (p. 17).

Dicho de otro modo, la globalización no sería un fenómeno exclusivamente contemporáneo, fruto del avance tecnológico digital, sino que las culturas híbridas podrían reconocerse en América Latina por aspectos como el mestizaje o el sincretismo religioso, hechos sociales muy anteriores pero no por eso menos vigentes.

Esta mirada puede llevarse a la interpretación de productos culturales mediáticos actuales. Por ejemplo, en el rock \& roll y el heavy metal, estilos musicales nacidos en Estados Unidos pero que han tenido casos notables de producción y difusión latinoamericana, como el movimiento de la Nueva Ola en Chile y bandas como Rata Blanca en Argentina, respectivamente. Más allá de sus connotaciones de homenaje (p.e. muchos cantantes de la Nueva Ola usaron seudónimos e incluso cantaron en inglés; el sonido y la voz de Rata Blanca fácilmente tributan a bandas anglo- sajonas como Iron Maiden), estas expresiones culturales populares, más que imitaciones, dan cuenta de una hibridación de identidades que se advierte por ejemplo en la validación de ambas en sus respectivos mercados locales.

Pero ¿qué elementos configuran esta hibridación? ¿Qué aspectos pueden reconocerse como de una y otra cultura? En este punto la lectura de Renato Ortiz parece más útil para el análisis.

La premisa de Ortiz (2004 [1994]) es la existencia de procesos globales que trascienden los grupos, las clases sociales y las naciones, en el marco de una sociedad global. Para él, con el cambio de milenio se hizo evidente que los seres humanos se encuentran "interligados, independientemente de sus voluntades" (p. 17), es decir, que somos ciudadanos del mundo pero no en el significado cosmopolita del viajero sino en uno cultural, más amplio y a la vez complejo, pues ha sido el mundo el que ha llegado hasta las personas, penetrando en su cotidiano.

La hipótesis es que "la mundialización dela cultura se revela a través de lo cotidiano" (ORTIZ, 2004, p. 18): la alimentación estandarizada (p.e. McDonald's) o que comparte patrones cuasi-ideológicos (p.e. el veganismo) o de aspiración identitaria (p.e. el auge de la dieta mediterránea) y la vestimenta regida por cánones internacionales explícitos (p.e. la industria medial en torno a la moda) e implícitos(p.e. la oferta de los centros comerciales, limitada a los dictámenes de la moda), son casos.

Ortiz (2004, pp. 31-33) critica los enfoques globalizadores tradicionales por su foco en los aspectos de la globalización económica y la suposición de que las demás esferas de la vida humana, incluyendo la cultura, están supeditadas a ella, y por la preeminencia de enfoques sistémicos estructuralistas, que dejan de lado al sujeto humano, agente cultural por excelencia (pp. 33-35). A partir de esta distinción construye su propia lectura: el término global(ización) 
lo usa para referirse a procesos económicos y tecnológicos, reservando mundial(ización) para los procesos culturales (p. 37).

La categoría mundo, entonces, incluye a lo global pero se expande al sumar la visión de mundo, el universo simbólico de la civilización: "Por eso prefiero decir que el inglés es una lengua mundial. Su transversalidad revela y expresa la globalización de la vida moderna; su mundialidad preserva a los otros idiomas en el interior de este espacio transglósico" (ORTIZ, 2004, p. 38).

Para Ortiz el mundo no es realmente sistémico"l' pues no es posible explicar su diversidad de fenómenos aplicando esquemas de distinción $n^{1 v}$, sino que más bien es un proceso y totalidad, es decir, algo que se reproduce y se deshace incesantemente "en el contexto de las disputas y de las aspiraciones divididas de los actores sociales, pero que se reviste [...] de una dimensión abarcadora, englobando otras formas de organización social: comunidades, etnias y naciones" (ORTIZ, 2004, p. 38).

Esta lógica de totalidad no significa que exista una cultura-mundo en el sentido estructuralista, pues eso implicaría tanto dicotomía como jerarquía (frente a otras culturas menores); la clave es que la mundialización impregna el conjunto de los fenómenos culturales, pues "una cultura mundializada corresponde a una civilización cuya territorialidad se globalizó" (ORTIZ, 2004, p. 39).

En este marco, el concepto de cultura-mundo no significa homogeneización cultural; para Ortiz este es un prejuicio adoptado desde la formulación de la sociedad de masas y retomado luego por la globalización del consumo — del cual McDonald's y el llamado Índice Big Mac pueden ser un ejemplo-, aunque él reconoce que la estandarización derivada del industrialismo ha penetrado ámbitos de la vida cultural (p.e. los modos de producción y de consumo), afectando nuestra percepción del fenómeno globalizador.
A esto se vincula la distinción entre patrón (o pattern, llamado por Ortiz patronización) y estándar (standard): el primero es un modo común de desarrollo que de todas formas admite diferencias entre casos, como una herencia, mientras que el segundo hace hincapié en la equivalencia de los casos, en su estatus de igualdad. En ese sentido, "una civilización promueve un patrón cultural sin con eso implicar la uniformización de todos. Una cultura mundializada segrega también un pattern, que yo calificaría de modernidad-mundo" (ORTIZ, 2004, pp. 41-42). En consecuencia, la modernidad-mundo apunta al conjunto de referentes generados por la mundialización de la cultura moderna.

Para ejemplificar los efectos de la mundialización en la interpretación de la realidad social Ortiz se sirve del caso de un ciudadano alemán que se siente perdido en la zona interior de China pero en casa estando en Hong Kong, en el entendido de que esta ciudad es más occidental. A este efecto le llama desterritorialización (ORTIZ, 2004, p. 113), pues corresponde a un espacio vacío de contenidos particulares, lo que también podría visualizarse en las ciudades-resort y las tiendas comerciales de los aeropuertos, que "parecen constituir una especie de no-lugares, locales anónimos, serializados, capaces de acoger a cualquier transeúnte, independientemente de su idiosincrasia" (p. 113).

Ahora bien, Ortiz advierte que estos no-lugares pueden llevar a pensar que es cada vez más difícil encontrarse, reconocerse en la identidad del lugar, pero eso no sería tan cierto si se considera que la mundialización de la cultura apunta precisamente a la existencia de referentes comunes. En otras palabras, la eficacia del resort como no-lugar se basa no en una pérdida de identidad sino en la sumatoria de identidades, pues busca ser un cualquier-lugar.

La desterritorialización no puede concebirse solo como abstracto, pues el espacio en sí es un concepto social, que adquiere 
sentido en el marco de la sociedad. Por eso busca localizarse llenando espacios mediante objetos mundializados (p.e. Coca Cola, McDonald's, Sony, etc.) que pueden encontrarse en todas las grandes urbes. Esto significa que "la mundialización no se sustenta sólo en el avance tecnológico, sino que hay un universo habitado por objetos compartidos a gran escala. Son ellos los que constituyen nuestro paisaje, amueblando nuestro medio ambiente" (ORTIZ, 2004, p. 114).

Asimismo, la desterritorialización se manifiesta por ejemplo en los modos de producción global: un auto alemán que se ensambla en Corea del Sur se vende en Chile; un filme estadounidense financiado por japoneses muestra locaciones de todo el mundo y se proyecta en todas partes. Esta deslocalización de la producción no solo tiene implicancias económicas (p.e. financieras y laborales) sino también sociales: no hay cómo definir el origen de las cosas ${ }^{\vee}$, y entonces los procesos de creación son selectivos, eligen partes de todo el mundo para construirse (ORTIZ, 2004, p.117). Aquí está la base de lo que él llama cultura internacional-popular (p. 118), que se manifiesta por ejemplo en la desterritorialización de la publicidad o del cine.

El propio Ortiz ofrece un ejemplo de la desterritorialización y cultura internacional-popular en las industrias mediales: el género fílmico del western. Surgido como representación de la colonización del territorio meridional y occidental estadounidense, incluyendo aspectos culturales propios del ser americano (p.e. la idea de una colonización financiada con recursos privados), su composición visual ${ }^{\mathrm{VI}}$ pudo ser captada y adoptada exitosamente por otras industrias fílmicas como la italiana, lo que en primera instancia se nota en las locaciones(desierto, montaña) pero además en el argumento (colonización, presencia del héroe solitario, enfrentamiento bien/mal), lo que desterritorializa el género. Ahora bien, esa extensión del western no solo se da en el cine: hay también expresiones en la moda (p.e. los jeans, llamados coloquialmente vaqueros), la televisión (p.e. Bonanza) y los imaginarios asociados a marcas de productos de consumo (p.e. Marlboro).

Ortiz (2004, p. 123) reconoce que este enfoque parece opuesto a la existencia de culturas nacionales, sobre todo porque existen estructuras sociales e instituciones cuya finalidad es la preservación y socialización de aspectos de cultura nacional, como el idioma, las tradiciones y los símbolos. Sin embargo, plantea que la idea de nación es algo reciente y que la formación de la identidad en las personas no depende de factores exclusivamente nacionales, no al menos en el mundo globalizado, al cual incluso los estados desean integrarse.

Otro ejemplo: la modernización industrial y la sociedad de consumo surgida desde mediados del siglo $X X$ no respetó fronteras nacionales (ORTIZ, 2004, p. 130) y tuvo impactos notables en la cultura nacional (p.e. al aumentar los índices de educación para acceder al mundo urbano y laboral). $Y$ en esto luego influyeron los medios y géneros masivos como la publicidad y el cine, que configuraron una memoria internacional-popular (p. 132), creando los referentes actuales para la modernidad-mundo.

Aquí hace referencia al concepto de Peter Berger de los universos simbólicos, que ordenan la historia localizando los eventos en una secuencia que incluye el pasado, el presente y el futuro: "En relación con el pasado establecen una memoria, compartida por los componentes de una colectividad; con respecto al futuro, definen un conjunto de proyecciones, modelos para las acciones individuales" (ORTIZ, 2004, p. 137). En resumen, el principal efecto de la cultura internacionalpopular es la generación de una memoria colectiva que reflexivamente valida y sustenta los patrones de su autoproducción. 


\subsection{Patronización del western}

Como se ha visto, para Ortiz las civilizaciones promueven patrones culturales sin necesariamente implicar homogeneización, pues la cultura opera más bien como un conjunto de referentes generados por la mundialización. En ese contexto explica la influencia de los bienes simbólicos producidos por las industrias culturales que, como en el caso de western, tendrían efectos en diversos ámbitos de la vida social, como el consumo y las relaciones sociales. Pero ¿qué patrones definen al western como género?

Grant (2007, p. 1) define al cine de género como aquellos filmes de factura comercial que, a través de la repetición y la variación de elementos, cuentan historias recurrentes sobre personajes reconocibles en contextos familiares. Esto puede observarse en tres niveles: uno de sistema general, en el cual diversos géneros se distinguen entre sí (p.e. western versus terror); otro de ámbito individual, en el que se analizan las características propias del género (p.e. la convención temporal clásica del western como situado entre mediados $y$ fines del siglo $X I X)$, $y$ otro del filme como producto individual (p. 2).

Para Grant (2007, p. 10) diversos elementos configuran un género. Por ejemplo, la existencia de convenciones estilísticas y narrativas, tanto del medio en que se produce el texto como del género mismo. Un caso en el cine es el punto de vista de la cámara, que equivale a un cuarto muro, y por eso los actores, salvo excepciones humorísticas o usos de cámara subjetiva, nunca miran ni hablan a la cámara. En el caso del western, las convenciones estilísticas incluyen por ejemplo el uso de una determinada tipografía (Wild West Font) en los créditos iniciales, así como el rodaje en exteriores. Otro elemento configurador de los géneros es la iconografía, entendida como el empleo de símbolos de uso general que son resignificados en el marco del relato de género
(GRANT, 2007, pp. 11-12), principalmente por la repetición. En el western serían por ejemplo el caballo, la diligencia, el revólver y las puertas batientes de las cantinas.

Un tercer elemento es el escenario (setting) o conjunto de atributos temporales y espaciales que dan vida al relato de género, en algunos casos además caracterizándolo (GRANT, 2007, p. 14). Esta variabilidad se aprecia al comparar géneros: la comedia romántica, por ejemplo, puede desarrollarse en casi cualquier momento y lugar, mientras que en el western el escenario canónico es el período del salvaje oeste (1865 a 1890) y el territorio conocido como la frontera americana (entre el río Mississippi y la costa del Pacífico).

Las historias y temas son también definitorias del cine de género (GRANT, 2007, pp. 15-16), sobre todo en los casos donde el relato de acción o cambio de estado concluye con un enfrentamiento, como sucede en el western clásico o en los filmes de terror. Dichas situaciones pueden asociarse con los aspectos de escenario; en el western, la pelea final suele ocurrir en la calle principal del pueblo, que entonces además adquiere valor iconográfico.

La definición de personajes es otro elemento (GRANT, 2007, pp. 17-18): no solo siguiendo estructuras actanciales (p.e. GREIMAS, 1987) o mitológicas (CAMPBELL, 1959), en el cine de género es relativamente más fácil reconocer a los agentes principales mediante caracterizaciones (p.e. en el western, sombreros blancos o negros según si el personaje es bueno o malo) y conductas estereotipadas (p.e. el machismo del gangster italoamericano).

Barry Langford (2005, p. 53), en tanto, valora al western como el primero de los cuatro grandes géneros clásicos del cine hollywoodense, junto al musical, al bélico y al de gangsters. Para él, el western puede ser considerado clave para la interpretación 
de la identidad estadounidense, por ejemplo respecto de mitos propios de la frontera americana que podrían replicarse en la relación de Estados Unidos con el mundo, como sucede con el uso de la violencia ritualizada y justificada (pp. 54-55).

En este punto, Langford (2005, p. 63) sigue al historiador Frederick Jackson Turner al sostener que el imaginario de la frontera que se advierte en el cine western no la muestra como un límite físico estático sino como un espacio móvil e indefinido de territorios por colonizar, es decir, una frontera móvil; el esfuerzo real, y a veces casi mitológico, por la pacificación de la frontera habría generado un carácter nacional estadounidense, menos identificado con los conflictos urbanos propios de la Europa industrializada y más propenso al choque entre la civilización y la naturaleza salvaje. Asimismo, la conquista del Oeste habría reducido las presiones sociales de aquellos grupos en situación de riesgo (p.e. los pobres o los inmigrantes), que en muchos casos se lanzaron a la aventura colonizadora por la promesa de forjar su propio des- tino, algo que también podría interpretarse como un reflejo del imaginario sobre la democracia estadounidense (p. 63).

Finalmente, McMahon y Csaki (2010, pp. 3-4) postulan que así como la soledad y la propensión a la aventura y la acción pueden ser consideradas las principales características del héroe canónico del western, al mismo tiempo dichas condiciones darían cuenta del auge del liberalismo y el pragmatismo durante el siglo XIX, encarnados en un ideal de masculinidad que se asocia a la propiedad de tierras o de dinero (p. 5), más que a la pertenencia a una comunidad.

\section{Caso de estudio: "Sal” (2012)}

Como se ha visto, el género western fue considerado por el propio Ortiz como referencia para explicar algunas formas de la cultura internacional-popular y la desterritorialización. En ese marco, un filme de este tipo producido en América Latina (véaseTabla 1) bien puede servir como caso de estudio.

\begin{tabular}{|c|c|}
\hline \multicolumn{2}{|r|}{ FICHA TÉCNICA DEL FILME "SAL" } \\
\hline Duración & 114 minutos \\
\hline Formato & $35 \mathrm{~mm} /$ Color \\
\hline Idioma & Español \\
\hline País & Chile \\
\hline Casa productora & Picardia Films (Chile), Cruz del Sur Cine (Argentina) \\
\hline Director & Diego Rougier (Argentina) \\
\hline Guion & Diego Rougier (Argentina) \\
\hline Productores ejecutivos & $\begin{array}{l}\text { Javiera Contador, Diego Rougier, Adrián Solar, Mario E. Levit, } \\
\text { Leonardo Hussen, Luigi Araneda }\end{array}$ \\
\hline Rodaje & Desierto de Atacama (Chile), Barcelona (España) \\
\hline Estreno nacional & $\begin{array}{l}2011 \text { (Festival Internacional de Cine de Viña del Mar); } \\
17 \text { de mayo de } 2012 \text { (Estreno en salas) }\end{array}$ \\
\hline Estreno internacional & 2012 (WorldFest de Houston, Estados Unidos) \\
\hline Actores principales & $\begin{array}{l}\text { Fele Martínez (España), Patricio Contreras (Chile), Javiera Contador } \\
\text { (Chile), Sergio Hernández (Chile), Luis Dubó (Chile), } \\
\text { Gonzalo Valenzuela (Chile) }\end{array}$ \\
\hline
\end{tabular}

Tabela 1 - Ficha técnica del filme "Sal"

Fuente: http://www.cinechile.cl/pelicula-1382 
"Sal" muestra la historia de un cineasta español llamado Sergio (Fele Martínez), fanático del género western que no consigue financiamiento para realizar su proyecto, ambientado en el Desierto de Atacama, en Chile. Las primeras secuencias lo muestran en Barcelona, en diversas reuniones con productores y amigos cuyas críticas al guion se centran en la carencia de conflictos atractivos y la falta de definición de los personajes, así como en la ausencia de motivos para filmar en el Desierto de Atacama (una verdadera obsesión de Sergio, que siempre justifica con lugares comunes, como la idea de que es "el desierto más árido del mundo"), lo que haría que el rodaje fuese más caro y menos atractivo para la industria española. Frustrado por los rechazos, Sergio decide viajar a Chile para elegir locaciones y encontrar la inspiración que necesita para acabar con su guion.

Al llegar a un pueblo (nunca identificado, pero por las locaciones se advierte que es Pica, en la Región de Tarapacá) en el Desierto de Atacama, Sergio es confundido por diversas personas con un tal Diego. De hecho, una anciana, creyendo que es Diego, le dice que no hay problema en que haya vuelto al pueblo porque "a Víctor le está yendo muy bien, así que ni se aparece por estos lados". Sergio no entiende nada, pero de todos modos la anciana lo invita a una fiesta que dará esa noche en su casa. Cuando ya es de tarde, ya gobiado por su bloqueo para continuar con la reescritura de su guion, Sergio decide ir a la fiesta para conocer algo de la realidad local, pero en el lugar todos vuelven a llamar lo Diego y lo felicitan por haber regresado.

De pronto uno de los invitados, llamado Pascua I (Luis Dubó), al darse cuenta de que es (o sería) Diego, lo mira con un gesto amenazante. Tras regresar a la pensión donde alojaba, Sergio es secuestrado por un grupo de hombres liderado por Pascual, quienes lo suben a una camioneta y lo llevan a un despoblado, donde conoce a Víctor (Patricio Contreras), líder del grupo, que también lo confunde con Diego. Luego de unas amenazas, Sergio le pide a Víctor aclarar la confusión, ante lo cual éste le pasa un revólver a Pascual y le ordena matarlo. Sin embargo el arma estaba descargada y todo fue un ardid para torturar a Sergio. Entonces Víctor les ordena a sus hombres llevar a Sergio a la casa del Viejo Vizcacha. Al amanecer, Sergio y sus secuestradores llegan a un rancho pobre en pleno desierto. Allí se lo entregan al Viejo Vizcacha (Sergio Hernández), un sujeto solitario que aparentemente forma parte de la red delictual de los secuestradores.

En los días siguientes Sergio establece una relación primero distante pero luego de complicidad con el Viejo Vizcacha, quien lo obliga a trabajar para poder comer. En sus conversaciones Sergio se entera de que Vizcacha también es una suerte de prisionero de Víctor, y que no huye del lugar porque allí están sepultados su esposa e hijo.

Una mañana los hombres de Víctor llegan al rancho a avisarle a Sergio (siempre confundido con Diego) que al día siguiente lo llevarían a la frontera con Bolivia para que participara en una internación ilegal de droga, con unos contactos de Pascual. Esa noche, mientras Sergio y el Viejo Vizcacha cenaban frente a una fogata, llega a la granja María (Javiera Contador), quien sin mediar explicaciones toma a Sergio, lo lleva a la casucha de Vizcacha y tiene un encuentro sexual con él. María también lo confunde con Diego y le pregunta si volvió para llevársela, tal como había prometido antes; entonces Sergio se entera de que ella es la esposa de Víctor, quien no solo lidera una banda de delincuentes 
sino que también es el hombre más poderoso del lugar, pues entre otras cosas soborna a la policía.

Al volver María a su jeep, el Viejo Vizcacha la mira de reojo y le dice "Puta", a lo que ella responde "Sí, pero no tuya". La escena siguiente muestra a María llegando a su casa y a Víctor oculto en la oscuridad, observándola.

Al día siguiente Sergio ensilla un caballo y parte a encontrarse con María en una antigua instalación minera en el desierto. Intentan escapar en el jeep de ella, pero en pleno desierto son interceptados por vehículos de la banda de Víctor. La escena siguiente muestra a Sergio colgado de un pozo, atado con una cadena, y a Víctor diciéndole que no puede confiar en una mujer, menos si es su mujer. Entonces les ordena a sus hombres soltar a Sergio, pero es solo para que ellos lo sometan: Víctor le corta el meñique de la mano izquierda. Tras eso Pascual y Héctor (Gonzalo Valenzuela), uno de los principales matones de Víctor, llevan a Sergio en una camioneta a un lugar en medio del desierto, lo golpean y lo dejan para que muera. Pascual le explica que "este es el desierto más árido del mundo; aquí no hay nada, solo sal y soledad", luego de lo cual le indica el camino para cruzar el salar... si es que puede. Tras golpearlo nuevamente, Héctor le quita las botas a Sergio y lo deja tirado.

Sergio camina por el desierto, sangrando por los golpes y las heridas en sus pies, mientras recuerda las críticas que productores y amigos le hicieron a su guion, sobre todo respecto de la falta de conflicto y de verosimilitud de su relato; luego alucina con Barcelona y con escenas de su película. Finalmente llega a una cabaña pobre y se desploma, extenuado; lo recoge una familia que había conocido la noche de la fiesta.
En su delirio, Sergio se imagina vestido de vaquero, disparándoles a los productores que rechazaron su proyecto. De pronto despierta en la casa del Viejo Vizcacha, quien le recomienda que deje de quejarse y decida vengarse.

Un par de días después Héctor va en su camioneta a buscar a Sergio y a Vizcacha. Mientras Sergio se sube a la camioneta, el Viejo se queda en su casucha, por lo que Héctor le dispara varias veces. Tras un tiroteo, Vizcacha logra matar a Héctor. Sergio y Vizcacha llevan el cuerpo de Héctor en su camioneta al desierto, donde lo abandonan. A la mañana siguiente, Vizcacha le enseña a Sergio a disparar. En el intertanto, Víctor y su banda encuentran el cadáver de Héctor, tras lo cual van al rancho de Vizcacha a preguntar qué pasó. Pascual amenaza a Sergio diciéndole que al día siguiente pasarían a buscarlo, y que aquel sería su viaje de despedida.

Esa noche, Sergio y el Viejo Vizcacha discuten qué hacer. Finalmente el anciano le dice a Sergio que debe enfrentarlos, empezando por Pascual. Sergio va a la casa de Julio (Jaime Omeñaca), donde Pascual usualmente cena porque está interesado en su hija adolescente. Tras un tiroteo en que Sergio hiere a Pascual, este muere asesinado por Julio. Al día siguiente los hombres de Víctor van a buscar a Sergio y lo llevan a la frontera, para la internación de droga desde Bolivia. Durante el viaje, Sergio golpea al conductor de la camioneta, que se desbarranca; solo él sobrevive. Al mismo tiempo Víctor y sus hombres van al rancho del Viejo Vizcacha. Al atardecer, Sergio logra regresar al rancho y encuentra a Vizcacha muerto; lo sepulta al lado de su esposa e hijo.

Al día siguiente Sergio va armado a la hacienda de Víctor. Tras matar a todos sus hombres, discute con él y 
lo enfrenta en un duelo con revólveres. Al verlo, Víctor le dice: "Ese revólver ya quiso matarme una vez, pero no pudo". "Lo sé", responde Sergio, "por eso estoy aquí". Al llegar el momento de batirse, Víctor dispara primero pero su arma no tenía balas; Sergio tiene tiempo para apuntar y dispararle en el estómago. "Yo sabía que íbamos a terminar así, de esta manera”, le dice Víctor. "¿Ha valido la pena?”, pregunta Sergio. "Sí, claro que sí", responde Víctor.

Cuando Sergio se va, Víctor le pide que no lo deje así, abandonado en el piso, y que le "regale" una bala, implicando que se suicidaría. Sin embargo, cuando Sergio se alejaba, Víctor intentó dispararle por la espalda. Se oye un disparo, pero no hiere a Sergio: Víctor había muerto. Tras esto Sergio toma su caballo y pretende irse, pero María lo detiene y le pide que se quede con ella, a lo que él responde que ya tiene una historia para contar. Mientras él se va, ella le grita que no puede irse, que habían acordado estar juntos. Él la ignora y sigue su marcha.

La secuencia final muestra a Sergio caminando junto a una ruta en pleno desierto, con su guion en la mano. Sergio recuerda al Viejo Vizcacha diciéndole "La sal lo come todo. La sal protege, pero también destruye. Corrompe todo lo que se le cruza en el camino. $Y$ nosotros estamos en medio de un salar". De pronto pasa un camión y Sergio le pide un aventón, pero el vehículo sigue de largo. Él se queda mirándolo, y se oye un disparo. Sergio advierte que está sangrando: María le había disparado por la espalda. "Pensaste que podías volver a dejarme", le dice ella. Él se desploma y el viento se lleva las hojas de su guion.

La toma siguiente muestra al teléfono de su departamento en Barcelona sonando; al activarse el buzón de voz, uno de sus amigos le cuenta que le ha conse- guido una cita con productores extranjeros. Al morir, Sergio se imagina la escena final de su película, con los antagonistas (él mismo y Víctor) riendo. María se aleja en su caballo, hacia el atardecer.

\section{Análisis: desterritorialización y iden- tidades}

Es posible advertir en "Sal" diversas referencias explícitas al género western, de acuerdo a los patrones descritos anteriormente. La historia misma es un relato sobre la producción de un filme de este género. Los créditos iniciales emplean una tipografía WildWest. El escenario de desarrollo de la historia, con matices propios dados por el Desierto de Atacama (p.e. parajes altiplánicos), muestra una zona fronteriza donde la institucionalidad jurídica y la policía no existen o son simplemente sobrepasadas por fuerzas fácticas; esta condición salvaje le otorga al territorio representado un aspecto de frontera móvil con efecto cultural, representado por ejemplo en la actitud del Viejo Vizcacha frente al abuso de Víctor y sus hombres: hay que vengarse, hay que matarlos.

El filme aprovecha también cierta iconografía canónica del género, como el uso de revólveres (si bien se emplean otras armas, el duelo final entre Sergio y Víctor es con estetipo), caballos (solo en el protagonista, pues los antagonistas se movilizan en camionetas), botas y sombreros vaqueros (presentes en casi todos, pero con significado especial para Sergio, pues operan primero como un simple disfraz pero luego como un activador de su nueva identidad de vaquero-justiciero). El duelo final entre Sergio y Víctor, aunque no se produce en la calle principal del pueblo, ocurre en un largo sendero interior de una hacienda, lo que les permite colocarse frente a frente $y$, por cierto, le permitió al director 
emplear tiros de cámara propios de las películas del género (p.e. planos americanos, contraplanos, planos de detalle al revólver y primeros planos cerrados de los duelistas).

En este sentido, la composición visual -o formato imagético, como diría Ortiz-del filme tributa en diversas oportunidades al canon del western: grandes planos generales del desierto y los protagonistas en movimiento (p.e. cuando Sergio cabalga para encontrarse con María; cuando ambos intentan huir en su jeep; cuando María camina hacia el atardecer, tras matar a Sergio); montaje cromático que privilegia los tonos cálidos del desierto y el atardecer (p.e. en la larga caminata de Sergio, tras ser abandonado por Pascual y Héctor; la toma final, de María cabalgando hacia el atardecer).

Finalmente, el protagonista es, no solo en su aventura por el desierto sino también en su vida en Barcelona, un tipo solitario: aunque en España lleva una vida aparentemente sin problemas, no se le conoce pareja ni familia; si bien su relación con María no es realmente amorosa, el hecho de que Sergio la deje al final de la película sin dudar e intente seguir su camino es también una referencia explícita al héroe clásico del género.

También se advierten referencias implícitas, como por ejemplo la musicalización del filme, encabezada por el tema central ("Ese revólver", compuesta e interpretada por Camilo Salinas), cuyas trompetas recuerdan la orquestación de Ennio Morricone para "Il buono, il brutto, il cattivo" ("El bueno, el malo y el feo", 1966, Sergio Leone) y, por extensión, a la de Dalparan y Jang Yeong-gyu para 좋은놈, 나쁜 $\square$, 이상한 $\square$ ("El bueno, el malo y el raro", 2008, Kim Jee-woon), otro caso de desterritorialización y cultura internacional-popular. Esta referencia al filme clásico de Leone se replica en el menú de la edición en DVD, donde la selección de escenas permite ver secuencias de los personajes principales, identificados con títulos como "El bueno" (Sergio), "El malo" (Víctor), "El feo" (Pascual), "La mujer" (María), "El lindo"(Héctor) y "El mentor" (Vizcacha).

¿Alcanzan estas características a constituir un producto cultural desterritorializado, de acuerdo con la conceptualización de Ortiz? En cierto modo sí, pues "Sal", no solo en su trama sino también en sus elementos estilísticos, busca localizarse mediante objetos mundializados. Sus permanentes referencias explícitas e implícitas al western son una manera de construirse como representante fiel del género. Incluso la referencia directa al Desierto de Atacama, potencialmente asociable solo a Chile, es siempre desde el argumento de su valoración internacional (que sería "el más árido del mundo").

Es decir, incluso la localidad del sitio de rodaje (p.e. Pica) consigue desterritorializarse, ya que finalmente las tomas del desierto operan casi como un no-lugar, algo que también podría decirse del Desierto de Tabernas (Almería, España), empleado por Sergio Leone para simular el territorio meridional de Estados Unidos, o por la zona occidental de China, elegida por Kim para simular el desierto de Manchuria. Con todo, el pueblo mismo no es retratado en su propia especificidad: nunca se dice que sea Pica, y solo algunas locaciones remiten a dicha localidad, por lo que perfectamente podría haberse rodado en más de un pueblo.

¿Qué identidades se presentan, entonces, en un filme desterritorializado como "Sal"?El protagonista, un español burgués que además es un artista ingenuo (p.e. porque desea vivir en un western pero nunca ha usado un arma ni conoce las locaciones con que sueña),opera 
como reflejo de la ácida lectura que Rougier entrega sobre Europa y su ensoñación delo latinoamericano como algo ajeno pero exótico y, por tanto, atractivo (incluso la obsesión de Sergio con el Desierto de Atacama podría explicarse por este motivo: Chile como el fin del mundo, la verdadera frontera).

Sergio es un hombre en principio unidimensional, sin historia ni entorno social salvo los productores y amigos (nunca se dice que lo sean; simplemente lo tratan con cercanía, pero eso puede ser frecuente en el mundo de la producción audiovisual) que critican su guion, pero durante su aventura adquiere matices y motivos diferentes, como la supervivencia y la venganza, tanto por el daño sufrido como por la muerte de su único aliado (Vizcacha).

El antagonista, Víctor, se asume que es de la zona (chileno) pero su acento tiene rasgos argentinos (probablemente una decisión de casting, pues es un sello de Patricio Contreras); su pasado solo se sugiere en relación al pasado de Diego, un sujeto que aparentemente lo habría traicionado, no solo al tener un amorío con su esposa sino también al violar ciertos códigos de lealtad masculina (no se dice, pero tal vez fue parte de su banda e incluso fue su amigo); su actitud ante el engaño de su mujer es violenta y vengativa, tanto con María como con Diego (Sergio), lo que da cuenta del machismo latinoamericano en general, sobre todo porque Víctor no tiene otra(s) mujer(es). Es decir, la ama y no está dispuesto a aceptar que ella lo deje ni que sus hombres (ni la comunidad local) se rían de él por ser un "cornudo" (de hecho, cuando Vizcacha se lo dice motiva que Víctor lo mate).

En consecuencia, las representaciones de identidad en "Sal" dan cuenta de una desterritorialización a-la-Ortiz, es decir, de la presencia de marcas de identidad no local sino global, lo que dificulta la definición identitaria del propio filme, convirtiéndolo en un objeto mundializado, un producto de la cultura internacional-popular. No por nada su estreno fue en un festival de cine y sus principales reconocimientos se han producido en el extranjero. Más que una película chilena, "Sal" exhibe una chilenización de lo internacional mediante guiños sutiles a lo local en un marco visual y argumental propio del western canónico.

\section{Discusión}

Este artículo presenta reflexiones en torno a la globalización en el ámbito cultural, a partir del análisis crítico de un filme que representa la hibridación de producciones simbólicas-mediáticas. El hecho de que la película pueda identificarse tan claramente con un género cinematográfico asociado a una identidad no-latinoamericana, pero que al mismo tiempo dé cuenta de ciertos aspectos identitarios de esta cultura, convierte a "Sal" en un caso de estudio válido. Pero existen muchos otros: las telenovelas y los reality shows (véase p.e. MAYER, 2003; TURNER, 2005),el romanticismo asociado a los vampiros y la figura ya universal de Drácula en la cultura popular (véase p.e. LIGHT, 2007; TAYLOR, 2014), o la transnacionalidad del hiphop (véase p.e. ALIM; IBRAHIM; PENNYCOOK, 2009; TICKNER, 2008), por mencionar algunos.

La lógica de la desterritorialización, que a su vez es una re-territorialización en la medida en que una pieza, un corpus o un imaginario se instalan en otra unidad de sentido -o son adoptados por ella, más bien-, es probablemente uno de los efectos culturales más evidentes de la globalización. La clave metodológica está, por tanto, en el reconocimiento 
de los patrones que permitan reconocer el género o el interdiscurso al cual dicha pieza o narrativa se acoplan.

Esa es, al mismo tiempo, la dificultad. En un contexto de hibridación, el reconocimiento de patrones, y sobre todo de géneros, es no solo complejo (multivariable) sino a veces inviable. Tal vez esa sea otro de los desafíos que nos impone la globalización.

\section{Bibliografía}

ALIM, H. Sami; IBRAHIM, Awad; PENNYCOOK, Alastair (eds.). Global linguistic flows: Hip-hop cultures, youth identities, and the politics of language. Nueva York: Routledge, 2009.

BAUMAN, Zygmunt. La globalización. Consecuencias humanas. Ciudad de México: Fondo de Cultura Económica, 2010.

CAMPBELL, Joseph. El héroe de las mil caras. Psicoanálisis del mito. Ciudad de México: Fondo de Cultura Económica, 1959.

CASTELLS, Manuel. La era de la información. Economía, sociedad y cultura. (Vol. 1, La sociedad red). Ciudad de México: Siglo XXI, 1999.

CASTLES, Stephen; DAVIDSON, Alastair. Citizenship and migration. Globalization and the politics of belonging. Londres: Macmillan, 2000.

CHAREONWONGSAK, Kriengsak. Globalization and technology: How will they change society? Technology in Society, ano 24, n. 3, 2002.

DEMONT-HEINRICH, Christof. Cultural imperialism versus globalization of culture: Riding the structure-agency dialectic in global communication and media studies. Sociology Compass, ano 5, n. 8, 2011.

GARCÍA CANCLINI, Néstor. Culturas híbridas. Buenos Aires: Paidós, 2001.

GARCÍA CANCLINI, Néstor. Lectores, espectadores e internautas. Barcelona: Gedisa, 2007.
GRANT, Barry Keith. Film genre. From iconography to ideology. Londres: Wallflower, 2007.

GREIMAS, Algirdas Julien. Semántica estructural. Investigación metodológica. Madrid: Gredos, 1987.

HALL, Stuart. Cultural identity and cinematic representation. Framework, n. 36, 1989.

HALL, Stuart; JEFFERSON Tony (eds.). Resistance through rituals. Youth subcultures in postwar Britain. Londres: Routledge, 1993.

INGLEHART, Ronald. Globalization and postmodern values. The Washington Quarterly, ano 23, n. 1, 2000.

JENSEN, Tommy; SANDSTRÖM, Johan. Stakeholder theory and globalization: The challenges of power and responsibility. Organization Studies, ano 32, n. 4, 2011.

KRAIDY, Marwan. Globalization of culture through the media. In: SCHEMENT, Jorge Reina (ed.), Encyclopedia of communication and information, Vol. 2. Nueva York: Macmillan Reference, 2002, P. 359-363.

KÜTTING, Gabriela. Globalization and the environment. Greening global political economy. Albany: State University of New York, 2004.

LANGFORD, Barry. Film genre. Hollywood and beyond. Edimburgo: Edinburgh University, 2005.

LIGHT, Duncan. Dracula tourism in Romania: Cultural identity and the state. Annals of Tourism Research, ano 34, n. 3, 2007.

LIZARDO, Omar. Understanding the flow of symbolic goods in the global cultural economy. International Journal of Contemporary Sociology, ano 45, n. 1, 2008.

LUHMANN, Niklas. Ecological communication. Chicago: University of Chicago, 1989.

LUHMANN, Niklas. Globalization or world society: How to conceive of modern society? International Review of Sociology, ano 7, n. 1,1997.

MAYER, Vicki. Living telenovelas/telenovelizing life: Mexican American girls' identities and transnational telenovelas. Journal of Communication, ano 53, n. 3, 2003.

MCLUHAN, Marshall; POWERS, Bruce R. La aldea global. Barcelona: Gedisa, 1995. 
MCMAHON, Jennifer; CSAKI, Steve. Introduction: Philosophy and the western. IN:MCMAHON, Jennifer; CSAKI, Steve (ds.), The philosophy of the western. Lexington: University of Kentucky, 2010, P. 1-10.

ORTIZ, Renato. Mundialización y cultura. Bogotá: Convenio Andrés Bello, 2004.

OUGAARD, Morten. Political globalization: State, power, and social forces. Nueva York: Palgrave Macmillan, 2004.

PARSONS, Talcott. The social system. Nueva York: The Free Press of Glencoe, 1959.

RITZER, George (Ed.). The Blackwell companion to globalization. Malden: Blackwell, 2007.

TAYLOR, Jessica. Romance and the female gaze obscuring gendered violence in the Twilight Saga. Feminist Media Studies, ano 14, n. 3, 2014.

TICKNER, Arlene. Aquí en el ghetto: Hip-hop in Colombia, Cuba, and Mexico. Latin American Politics and Society, ano 50, n. 3, 2008.

TURNER, Graeme. Cultural identity, soap narrative, and reality TV. Television \& New Media, ano 6, n. 4, 2005.

WILLIAMS, Raymond. Television: Technology and cultural form. Londres: Collins, 1974.
I Pablo Matus Lobos. Chile. Doutor em Ciencias de la Comunicación. Faculdad de Comunicaciones, Pontificia Universidad Católica de Chile. Contato: pmatus@uc.cl

Il Al menos en sus primeros acercamientos al tema, pues en sus últimas obras se muestra escéptico de la vigencia misma del concepto (García Canclini, 2007, pp. 9-11).

III A la usanza de las interpretaciones estructuralesfuncionalistas, como la de Talcott Parsons, o las directamente funcionalistas, como la de Niklas Luhmann.

IV Por ejemplo la identificación de prerrequisitos funcionales en Parsons (1959, pp. 26-35) y la codificación binaria de informaciones en Luhmann (1989, pp. 36-43).

V Ni potencialmente, de las ideas: por eso Ortiz se desterritorializa como analista.

VI Que Ortiz (2004, p. 121) llama formato imagético. 